\title{
Elliptic flow for $\varphi$ mesons measured by PHENIX
}

\author{
Iurii Mitrankov ${ }^{1, *}$, Yaroslav Berdnikov ${ }^{1}$, Alexander Berdnikov ${ }^{1}$, and Dmitry Kotov ${ }^{1}$ \\ ${ }^{1}$ Peter the Great St.Petersburg Polytechnic University (SPbPU)
}

\begin{abstract}
The systematic study of hadronic elliptic flow in various relativistic heavy ion collisions is important for the investigation of the initial geometry influence on the quark gluon plasma characteristics. The $\varphi$ meson consists of strange and antistrange quarks and has a small interaction cross section with non-strange hadrons. Therefore, $\varphi$ mesons are barely affected by late hadronic stage and reflect detailed information about hot and dense matter properties. Additionally, the comparison of elliptic flow for $\varphi$ mesons to those of charged hadrons will provide additional information on the flavor dependence of flow. PHENIX has measured second order azimuthal anisotropy coefficients for $\varphi$ mesons in $\mathrm{Cu}+\mathrm{Au}$ collisions at $\sqrt{s_{N N}}=200 \mathrm{GeV}$ and in $\mathrm{U}+\mathrm{U}$ collisions at $\sqrt{s_{N N}}=193 \mathrm{GeV}$ at midrapidity $(|\eta|<0.35)$. The obtained data suggest scaling of elliptic flow for $\varphi$ mesons with eccentricity of participant nucleons in $\mathrm{Cu}+\mathrm{Au}, \mathrm{U}+\mathrm{U}$, and $\mathrm{Au}+\mathrm{Au}$ collisions. Viscous hydrodynamic model iEBE-VISHNU provides a simultaneous description of the obtained data.
\end{abstract}

\section{Introduction}

One of the most important goals of the current ultra-relativistic heavy ion research is the investigation of the quark gluon plasma (QGP) [1] properties. Study of second order azimuthal anisotropy has played a key role in the establishment of the QGP formation at the Relativistic Heavy Ion Collider [2]. Elliptic flow is commonly quantified by the second Fourier moment $v_{2} \equiv\left\langle\cos 2\left(\phi-\Psi_{R P}\right)\right\rangle$ of the azimuthal momentum distribution [3]. The detailed dependencies of $v_{2}$ on centrality, transverse momentum $p_{T}$, and particle species can lend insight on reaction dynamics and are important for defining parameters of viscous hydrodynamic, which may describe QGP behavior [4].

According to the Okubo-Zweig-Iizuka (OZI) rule $\varphi$-mesons have a relatively large mean free path, compared to the transverse size of the emitting system and those of $\pi^{ \pm}$-meson $[5,6]$. Also, the $\varphi$-mesons mostly decay after the QGP phase [1,5], therefore $\varphi$ kinematic properties are not affected by hadronic stage and bring detailed information of the QGP properties. Since the $\varphi$-meson mass is comparable to the (anti)protons $(p+\bar{p}) / 2$ mass, the comparisons of $\varphi$ meson, $\pi^{ \pm}$-meson and $(p+\bar{p}) / 2$ provide an investigation of $v_{2}$ dependence on quark content and hadron mass. If the hadronic $v_{2}$ is proportional to the number of valence quarks $n_{q}$ of the hadron, the flowing medium reflects quark degrees of freedom, otherwise hadronic stage is responsible for elliptic flow development [2].

The study of $\varphi$-meson production in heavy ion collisions, such as $\mathrm{Cu}+\mathrm{Au}$ and $\mathrm{U}+\mathrm{U}$ collisions, indicate additional mechanisms involved in its production in these collision systems

\footnotetext{
*e-mail: mitrankovy@gmail.com
} 
[7, 8]. Additionally, anisotropic flow is strongly coupled to the medium density, initial geometric shape, therefore $v_{2}$ for $\varphi$-mesons was studied in asymmetric $\mathrm{Cu}+\mathrm{Au}$ collisions and collisions of deformed uranium nucleus $\mathrm{U}+\mathrm{U}$.

To investigate the underlying processes behind $v_{2}$ evolution, the comparison of experimental elliptic flow $v_{2}$ for $\varphi$-mesons to theoretical predictions is needed. The iEBE-VISHNU model [9] performs event-by-event simulations for relativistic heavy-ion collisions using $(2+1) \mathrm{D}$ viscous hydrodynamic and hadronic cascade model. This model has proved itself valid for recent PHENIX results on elliptic and triangular flow for charged hadrons, published in the Nature Physics [10].

In this paper elliptic flow for $\varphi$-mesons in $\mathrm{Cu}+\mathrm{Au}$ collisions at $\sqrt{s_{N N}}=200 \mathrm{GeV}$ and in $\mathrm{U}+\mathrm{U}$ collisions at $\sqrt{s_{N N}}=193 \mathrm{GeV}$ is studied as a function of centrality and kinetic properties, i.e. transverse momentum $p_{T}$ and transverse kinetic energy $K E_{T}$. The obtained results are compared to iEBE-VISHNU hydrodynamic model predictions.

\section{Results and discussion}

The procedure for calculating reaction plane azimuthal angle in $\mathrm{Cu}+\mathrm{Au}$ collisions at $\sqrt{s_{N N}}=200 \mathrm{GeV}$ and in $\mathrm{U}+\mathrm{U}$ collisions at $\sqrt{s_{N N}}=193 \mathrm{GeV}, \varphi$ meson $v_{2}$, and systematic uncertainties can be found elsewhere [11-13].

The left and right panels of Figure 1 compare the unscaled and scaled (respectively) $\varphi$ meson $v_{2}\left(p_{T}\right)$ in $0-20 \%, 20-40 \%, 40-60 \%$, and $20-60 \% \mathrm{Cu}+\mathrm{Au}, 0-50 \% \mathrm{U}+\mathrm{U}$ collisions, previous 20-60\% Au+Au PHENIX results, 0-30\% and 30-80\% Au+Au STAR results [11]. The scaling of $\varphi$-meson elliptic flow suggests that the $v_{2}$ values follow common empirical scaling with $\varepsilon_{2} N_{\text {part }}^{1 / 3}$. The same scaling was observed in previous PHENIX results on charged hadrons $v_{2}$ in $\mathrm{Cu}+\mathrm{Au}, \mathrm{Au}+\mathrm{Au}$, and $\mathrm{Cu}+\mathrm{Cu}$ collisions at $\sqrt{s_{N N}}=200 \mathrm{GeV}$ [13]. The average number of participating nucleons $N_{\text {part }}$ and participant eccentricity of second order $\varepsilon_{2}$ were estimated using Glauber model Monte-Carlo simulation of the each collision system. Scaling with participant eccentricity of second order $\varepsilon_{2}$ represents dependence of $v_{2}$ on collision geometry. While $N_{\text {part }}$ is proportional to the volume of the QGP, $N_{\text {part }}^{1 / 3}$ is proportional to the radius of the QGP.
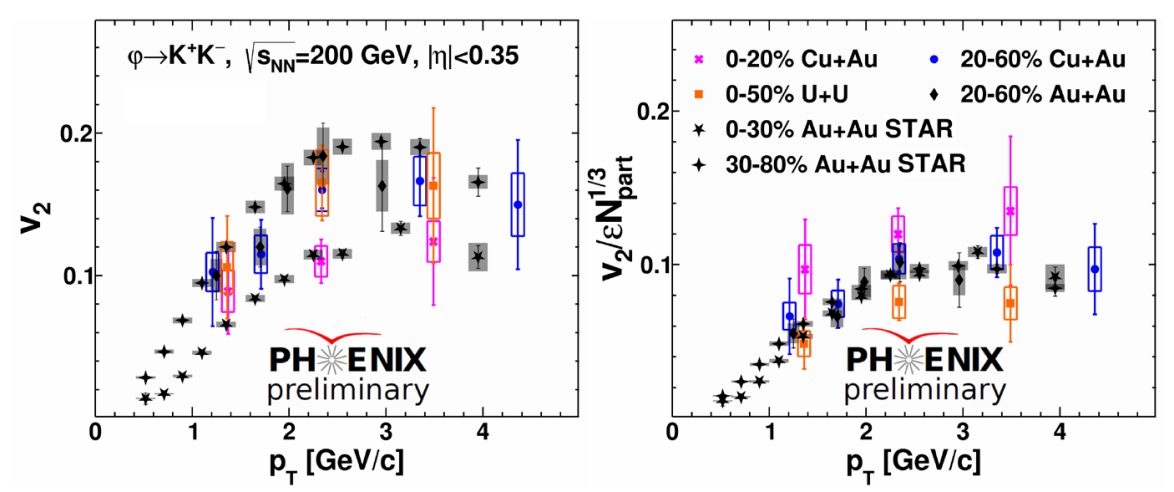

Figure 1. The elliptic flow $v_{2}$ (left) and $v_{2} /\left(\varepsilon_{2} N_{\text {part }}^{1 / 3}\right)$ (right) vs. $p_{T}$ for $\varphi$-mesons in $0-20 \%, 20-40 \%$, 40-60\%, and 20-60\% Cu+Au collisions, 0-50\% U+U collisions, previous 20-60\% Au+Au PHENIX results, $0-30 \%$ and $30-80 \% \mathrm{Au}+\mathrm{Au}$ STAR results. Here and below error bars and open boxes around points correspond to statistical and systematic uncertainties. 

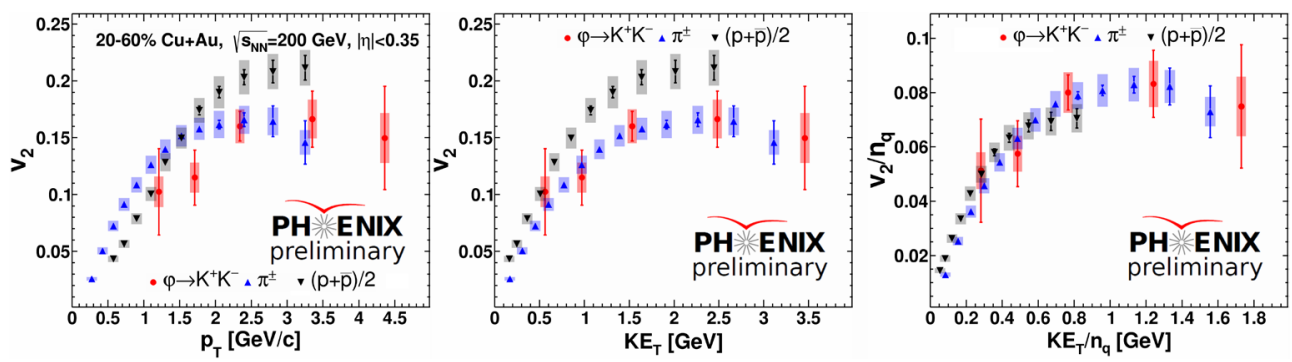

Figure 2. The comparison of elliptic flow $v_{2}$ and $v_{2} / n_{q}$ for $\varphi$-mesons in 20-60\% $\mathrm{Cu}+\mathrm{Au}$ collisions to those for $\pi^{ \pm}$and $(p+\bar{p}) / 2$ [14] as a function of $p_{T}$ (left), $K E_{T}$ (middle) and $K E_{T} / n_{q}$ (right).
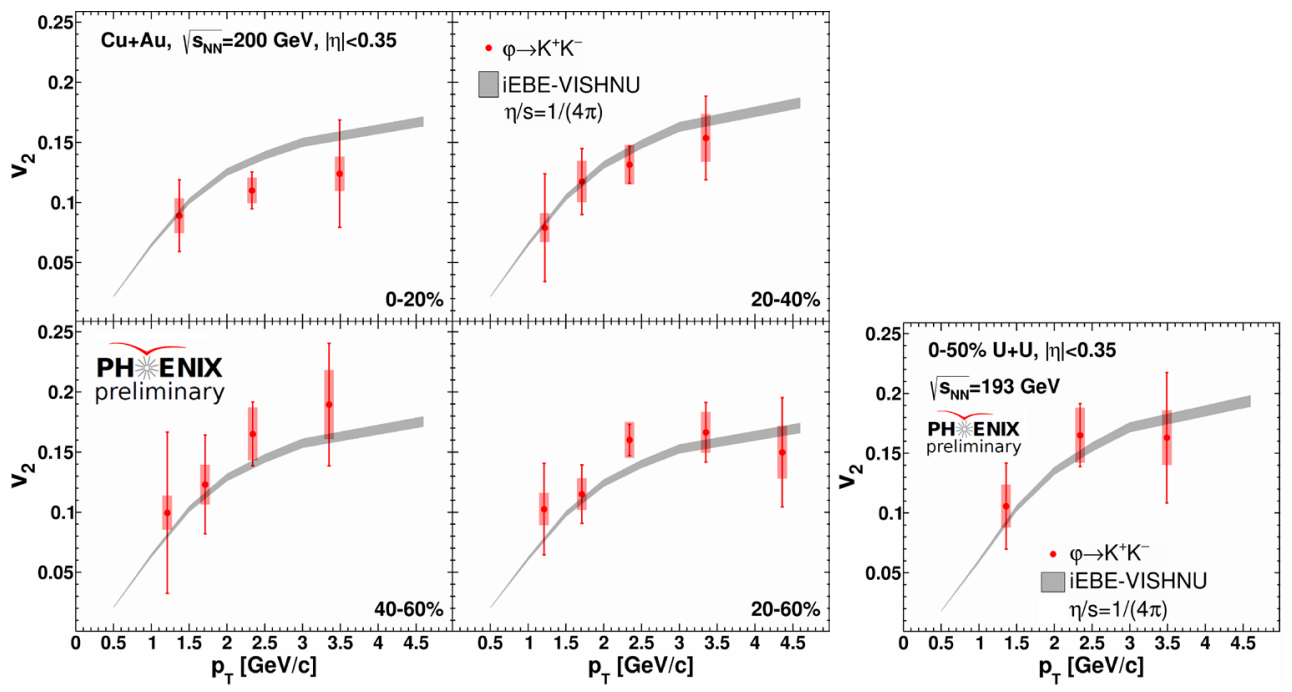

Figure 3. The comparison of measured elliptic flow $v_{2}\left(p_{T}\right)$ for $\varphi$-mesons in 0-20\%, 20-40\%, 40-60\%, and $20-60 \% \mathrm{Cu}+\mathrm{Au}$ collisions at $\sqrt{s_{N N}}=200 \mathrm{GeV}$ and in $0-50 \% \mathrm{U}+\mathrm{U}$ collisions at $\sqrt{s_{N N}}=193 \mathrm{GeV}$ to iEBE-VISHNU hydrodynamic model prediction with specific viscosity $\eta / s=1 /(4 \pi)$.

The example of the comparison of elliptic flow obtained for $\varphi$-mesons in 20-60\% Cu+Au collisions at $\sqrt{s_{N N}}=200 \mathrm{GeV}$ to those for $\pi^{ \pm}$and $(p+\bar{p}) / 2$ [14] is shown in Figure 2. The scaling of elliptic flow for light hadrons with the number of valence quarks $n_{q}$ of the hadron and transverse kinetic energy per one quark $K E_{T} / n_{q}$ is observed (Figure 2 right panel). This result along with a smaller rescattering cross section for $\varphi$-mesons than for $\pi^{ \pm}$may indicate that elliptic flow development occurs before hadronization in the QGP phase of heavy-ion collision.

In order to better understand the physics behind $v_{2}$ development, the comparisons of measured elliptic flow for $\varphi$-mesons in $0-20 \%, 20-40 \%, 40-60 \%$, and $20-60 \% \mathrm{Cu}+\mathrm{Au}$ collisions and in $0-50 \% \mathrm{U}+\mathrm{U}$ collisions to iEBE-VISHNU model predictions are shown in Figure 3. The iEBE-VISHNU model is based on $(2+1) \mathrm{D}$ viscous hydrodynamic and includes the QGP formation. The results are well described with $v_{2}$ obtained with iEBE-VISHNU calculations. A specific viscosity value of $\eta / s=1 /(4 \pi)$ was used in the calculations. 


\section{Conclusion}

Elliptic flows for $\varphi$-mesons were measured in $\mathrm{Cu}+\mathrm{Au}$ collisions at $\sqrt{s_{N N}}=200 \mathrm{GeV}$ and in $\mathrm{U}+\mathrm{U}$ collisions at $\sqrt{s_{N N}}=193 \mathrm{GeV}$ as a function of $p_{T}, K E_{T}$, and $K E_{T} / n_{q}$ and compared with previous measurements in Au+Au collisions, $v_{2}$ for $\pi^{ \pm}$and $(p+\bar{p}) / 2$, and hydrodynamic model iEBE-VISHNU calculations.

The scaling of obtained $\varphi$-meson $v_{2}$ with second order participant eccentricity, and cube root of the number of participant nucleons is observed and confirms the previous PHENIX results. Additionally, data suggest dependence of light hadron elliptic flow on the number of valence quarks $n_{q}$ of the hadron. Therefore, system size and geometry influence can be considered by normalizing factor $\varepsilon_{2} N_{\text {part }}^{1 / 3}$, whereas hadron type dependence - by scaling with $n_{q}$. Due to all above, elliptic flow development probably occurs before hadronization in the QGP phase of heavy-ion collision.

The agreement of experimental data with iEBE-VISHNU calculations suggests that QGP behaviour can be described with $(2+1) \mathrm{D}$ viscous hydrodynamic with specific viscosity $\eta / s=$ $1 /(4 \pi)$.

\section{Acknowledgments}

The research is partially funded by the Ministry of Science and Higher Education of the Russian Federation under the strategic academic leadership program 'Priority 2030' (Agreement 075-15-2021-1333 dated 30.09.2021)

\section{References}

[1] K. Adcox et al. (PHENIX), Nuclear Physics A 757, 184-283 (2005)

[2] S.S. Adler et al. (PHENIX), Phys. Rev. Lett. 94, 232302 (2005)

[3] S. Voloshin, Y. Zhang, Z. Phys. C 70, 665 (1996)

[4] V. Greco, C.M. Ko, P. Lévai, Phys. Rev. C 68, 034904 (2003)

[5] A. Shor, Phys. Rev. Lett. 54, 1122 (1985)

[6] P. Zyla et al. (Particle Data Group), PTEP 2020, 083 C01 (2020)

[7] A. Berdnikov, D. Kotov, I. Mitrankov, Journal of Physics: Conference Series 1135, 012044 (2018)

[8] M.M. Mitrankova, Y.A. Berdnikov, A.Y. Berdnikov, D.O. Kotov, I.M. Mitrankov (PHENIX), Phys. Scripta 96, 084010 (2021)

[9] C. Shen, Z. Qiu, H. Song, J. Bernhard, S. Bass, U. Heinz, The iEBE-VISHNU code package for relativistic heavy-ion collisions (2015)

[10] C. Aidala et al. (PHENIX), Nature Phys. 15, 214 (2019)

[11] S. Afanasiev et al. (PHENIX), Phys. Rev. Lett. 99, 052301 (2007)

[12] A. Adare et al. (PHENIX), Phys. Rev. C 88, 064910 (2013)

[13] A. Adare et al. (PHENIX), Phys. Rev. C 94, 054910 (2016)

[14] J. Barrette, R. Bellwied, S. Bennett et al., Phys. Rev. C 56, 3254 (1997) 